\title{
A Study in Improving BLEU Reference Coverage with Diverse Automatic Paraphrasing
}

\author{
Rachel Bawden $^{1}$ Biao Zhang ${ }^{1} \quad$ Lisa Yankovskaya $^{2} \quad$ Andre Tättar $^{2}$ Matt Post $^{3}$ \\ ${ }^{1}$ School of Informatics, University of Edinburgh, Scotland \\ ${ }^{2}$ University of Tartu, Tartu, Estonia \\ ${ }^{3}$ Johns Hopkins University, Baltimore, Maryland, USA
}

\begin{abstract}
We investigate a long-perceived shortcoming in the typical use of BLEU: its reliance on a single reference. Using modern neural paraphrasing techniques, we study whether automatically generating additional diverse references can provide better coverage of the space of valid translations and thereby improve its correlation with human judgments. Our experiments on the into-English language directions of the WMT19 metrics task (at both the system and sentence level) show that using paraphrased references does generally improve BLEU, and when it does, the more diverse the better. However, we also show that better results could be achieved if those paraphrases were to specifically target the parts of the space most relevant to the MT outputs being evaluated. Moreover, the gains remain slight even when using human paraphrases elicited to maximize diversity, suggesting inherent limitations to BLEU's capacity to correctly exploit multiple references. Surprisingly, we also find that adequacy appears to be less important, as shown by the high results of a strong sampling approach, which even beats human paraphrases when used with sentencelevel BLEU. ${ }^{1}$
\end{abstract}

\section{Introduction}

There is rarely a single correct way to translate a sentence; work attempting to encode the entire translation space of a sentence suggests there may be billions of valid translations (Dreyer and Marcu, 2012). Despite this, in machine translation (MT), system outputs are usually evaluated against a single reference. This especially affects MT's dominant metric, BLEU (Papineni et al., 2002), since it is a surface metric that operates on explicit $n$-gram overlap (see. (1) showing two ade-

\footnotetext{
${ }^{1}$ Our code and outputs are available at https:// github.com/rbawden/paraphrasing-bleu.
}

quate MT outputs, one with only minimal overlap with the reference): ${ }^{2}$

\section{(1) Ref: This did not bother anybody. $\mathrm{MT}_{1}$ : This didn 't bother anybody . $\mathrm{MT}_{2}$ : Nobody was bothered by this .}

Almost since its creation, BLEU's status as the dominant metric for MT evaluation has been challenged (e.g., Callison-Burch et al. (2006), Mathur et al. (2020)). Such work typically uses only a single reference, however, which is a deficient form of the metric, since one of BLEU's raisons d'être was to permit the use of multiple references, in a bid to represent "legitimate differences in word choice and word order." Unfortunately, multiple references are rarely available due to the high cost and effort of producing them. One way to inexpensively create them is with automatic paraphrasing. This has been tried before (Zhou et al., 2006; Kauchak and Barzilay, 2006), but only recently have paraphrase systems become good enough to generate fluent, high quality sentential paraphrases (with neural MT-style systems). Moreover, it is currently unclear (i) whether adding automatically paraphrased references can provide the diversity needed to better cover the translation space, and (ii) whether this increased coverage overlaps with observed and valid MT outputs, in turn improving BLEU's correlation with human judgments.

We explore these questions, testing on all intoEnglish directions of the WMT19 metrics shared task (Ma et al., 2019) at the system and segment level. We compare two approaches: (i) generating diverse references with the hope of covering as much of the valid translation space as possible, and (ii) more directly targeting the relevant areas of the translation space by generating paraphrases that contain $n$-grams selected from the system out-

\footnotetext{
${ }^{2}$ See Sec. 4.2 and (Papineni et al., 2002, §1.1) for details.
} 
puts. This allows us to compare the effects of diversity against an upper bound that has good coverage. We anchor our study by comparing automatically produced references against humanproduced ones on a subset of our data.

Our experiments show that adding paraphrased references rarely hurts BLEU and can provide moderate gains in its correlation with human judgments. Where it does help, the gains are correlated with diversity (and less so adequacy), but see diminishing returns, and fall short of the nondiverse method designed just to increase coverage. Manual paraphrasing does give the best systemlevel BLEU results, but even these gains are relatively limited, suggesting that diversity alone has its limits in addressing weaknesses of surfacebased evaluation metrics like BLEU.

\section{Related Work}

Paraphrasing for MT evaluation There is a long history of using paraphrasing to overcome the limitations of BLEU-style metrics. Some early approaches rely on external resources (e.g. WordNet) to provide support for synonym matching (Banerjee and Lavie, 2005; Kauchak and Barzilay, 2006; Denkowski and Lavie, 2014). More automatic methods of identifying paraphrases have also been developed. An early example is ParaEval (Zhou et al., 2006), which provides local paraphrase support using paraphrase sets automatically extracted from MT phrase tables. More recently, Apidianaki et al. (2018) exploit contextual word embeddings to build automatic HyTER networks. However they achieve mixed results, particularly when evaluating high performing (neural) models.

The use of MT systems to produce paraphrases has also been studied previously. Albrecht and Hwa (2008) create pseudo-references by using out-of-the-box MT systems and see improved correlations with human judgments, helped by the systems being of better quality than those evaluated. This method was extended by Yoshimura et al. (2019), who filter the pseudo-references for quality. An alternative strategy is to use MT-style systems as paraphrasers, applied to the references. Madnani et al. (2007) show that additional (paraphrased) references, even noisy ones, reduce the number of human references needed to tune an SMT system, without significantly affecting MT quality. However their aim for coverage over quality means that their paraphrases are unlikely to be good enough for use in a final evaluation metric.

Despite the attention afforded to the task, success has been limited by the fact that until recently, there were no good sentence-level paraphrasers (Federmann et al. (2019) showed that neural paraphrasers can now outperform humans for adequacy and cost). Attempts (e.g. Napoles et al., 2016) using earlier MT paradigms were not able to produce fluent output, and publicly available paraphrase datasets have only been recently released (Wieting and Gimpel, 2018; Hu et al., 2019a). Moreover, most works focus on synonym substitution rather than more radical changes in sentence structure, limiting the coverage achieved.

Structurally diverse outputs Diverse generation is important to ensure a wide coverage of possible translations. Diversity, both lexical and structural, has been a major concern of text generation tasks (Colin and Gardent, 2018; Iyyer et al., 2018). State-of-the-art neural MT-style text generation models used for paraphrasing (Prakash et al., 2016; Mallinson et al., 2017) typically suffer from limited diversity in the beam. Techniques such as sampling from the model distribution or from noisy outputs have been proposed to tackle this (Edunov et al., 2018) but can harm output quality.

An effective strategy to encourage structural diversity is to add syntactic information (which can be varied) to the generated text. The constraints can be specified manually, for example by adding a parse tree (Colin and Gardent, 2018; Iyyer et al., 2018) or by specifying more abstract constraints such as rewriting embeddings (Xu et al., 2018). A similar but more flexible approach was adopted more recently by Shu et al. (2019), who augment target training sentences with cluster pseudotokens representing the structural signature of the output sentence. When decoding, the top cluster codes are selected automatically using beam search and for each one a different hypothesis is selected. We adopt Shu et al.'s approach here, due to the automatic nature of constraint selection and the flexibility afforded by constraint definition, allowing us to test different types of diversity by varying the type of sentence clustering method.

\section{Generating paraphrased references}

We look at two ways to produce paraphrases of English references using English-English NMT architectures. The first (Sec. 3.1) aims for maximal lexical and syntactic diversity, in a bid to 
better cover the space of valid translations. In contrast, the second (Sec. 3.2) aims to produce paraphrases that target the most relevant areas of the space (i.e. that are as close to the good system outputs as possible). Of course, not all outputs are good, so we attempt to achieve coverage while maintaining adequacy to the original reference by using information from the MT outputs. While less realistic practically, this approach furthers the study of the relationship between diversity and valid coverage.

\subsection{Creating diverse paraphrases}

To encourage diverse paraphrases, we use Shu et al.'s (2019) method for diverse MT, which consists in clustering sentences according to their type and training a model to produce outputs corresponding to each type. Applied to our paraphrasing scenario, the methodology is as follows:

1. Cluster target sentences by some property (e.g., semantic, syntactic representation);

2. Assign a code to each cluster and prefix each target sentence in the training data with its code (a pseudo-token), as follows:

(2) $\langle$ cl_14 $\rangle$ They knew it was dangerous $\langle$ cl_101〉 They had chickens, too . $\langle$ cl_247 $\rangle$ That 's the problem .

3. Train an NMT-style paraphrase model using this augmented data;

4. At test time, apply the paraphraser to each reference in the test set; beam search is run for each of the $n$ most probable sentence codes to produce $n$ paraphrases per reference.

As in (Shu et al., 2019), we test two different types of diversity: semantic using LASER sentential embeddings (Artetxe and Schwenk, 2019) and syntactic using a TreeLSTM encoder (Tai et al., 2015). Both methods encode each sentence as a vector, and the vectors are clustered using $k$ means into 256 clusters (full details in App. C).

Semantic: We use pretrained LASER sentential embeddings (Artetxe and Schwenk, 2019) to encode sentences into 1024-dimensional vectors.

Syntactic: As in (Shu et al., 2019), we encode constituency trees into hidden vectors using a TreeLSTM-based recursive autoencoder, with the difference that we use $k$-means clustering to make the method more comparable to the above, and we encode syntactic information only.

\subsection{Output-guided constrained paraphrases}

Diversity is good, but even a highly diverse set of references may not necessarily be in the same space as the MT outputs. We attempt to achieve high coverage of the system outputs by using a weak signal from those outputs. The signal we use is unrewarded n-grams from the best systems, which are $n$-grams in system outputs absent from the original reference. We identify them as follows. For each sentence in a test set, we find all $n$-grams that are (a) not in the reference but (b) are present in at least $75 \%$ of the system outputs, (c) limited to the top half of systems in the human system-level evaluation (Barrault et al., 2019). Then, for each such $n$-gram, we generate one paraphrase of the reference using constrained decoding (Post and Vilar, 2018), with that $n$-gram as a constraint. This gives a variable-sized set of paraphrased references for each sentence. In order to limit overfitting to the best systems, we use a cross-validation framework, in which we randomly split the submitted systems into two groups, the first used to compute the $n$-gram constraints and the augmented references, and the second half for evaluation. We repeat this ten times and report the average correlation across the splits.

\section{Experiments}

Our goal is to assess whether we can generate paraphrases that are representative of the translation space and which, when used with BLEU, improve its utility as a metric. We therefore carry out experiments to (i) evaluate the adequacy and diversity of our paraphrases (Sec. 5.2) and (ii) compare the usefulness of all methods in improving BLEU's correlation with human judgments of MT quality (Sec. 4.1). BLEU is a corpus-level metric, and our primary evaluation is therefore its systemlevel correlation. However, it is often also used at the segment level (with smoothing to avoid zero counts). It stands to reason that multiple references would be more important at the segmentlevel, so we also look into the effects of adding paraphrase references for SENTBLEU too.

\subsection{Metric evaluation}

For each set of extra references, we produce multireference BLEU and SENTBLEU metrics, which we use to score all into-English system outputs from the WMT19 news task. ${ }^{3}$ We evaluate the

\footnotetext{
${ }^{3}$ http://statmt.org/wmt19/results.html.
} 
scores as in the metrics task (Ma et al., 2019), by calculating the correlation with manual direct assessments (DA) of MT quality (Graham et al., 2013). System-level scores are evaluated using Pearson's $r$ and statistical significance of improvements (against single-reference BLEU) using the Williams test (Williams, 1959). Segment-level correlations are calculated using Kendall's $\tau$ (and significance against single-reference SENTBLEU with bootstrap resampling) on the DA assessments transformed into relative rankings.

\subsection{Baseline and contrastive systems}

Our true baselines are case-sensitive corpus BLEU and SENTBLEU, both calculated using sacreBLEU (Post, 2018) using the standard BLEU formula. Though likely familiar to the reader, we review it here. BLEU is computed by averaging modified $n$-gram precisions $\left(p_{n}, n=1 . .4\right)$ and multiplying this product by a brevity penalty (BP), which penalizes overly short translations and thereby works to balance precision with recall:

$$
\begin{aligned}
\mathrm{BLEU} & =\mathrm{BP} \cdot \exp \left(\sum_{n=1}^{N} w_{n} \log p_{n}\right) \\
\mathrm{BP} & = \begin{cases}1 & \text { if } c>r \\
e^{1-r / c} & \text { if } c \leq r\end{cases} \\
p_{n} & =\frac{\sum_{h \in H} \sum_{\text {ngram } \in h} \# \text { clip } \text { (ngram) }}{\sum_{h^{\prime} \in H} \sum_{\text {ngram' } \in h^{\prime}} \# \text { (ngram') }},
\end{aligned}
$$

with $c$ and $r$ the lengths of the hypothesis and reference sets respectively, $H$ is the set of hypothesis translations, \# (ngram) the number of times ngram appears in the hypothesis, and \# clip $_{\text {(ngram) }}$ is the same but clipped to the maximum number of times it appears in any one reference.

By definition, BLEU is a corpus-level metric, since the statistics above are computed across sentences over an entire test set. The sentence-level variant requires a smoothing strategy to counteract the effect of $0 n$-gram precisions, which are more probable with shorter texts. We use exponential smoothing. Both baselines use the single provided reference only. We also compare against several contrastive paraphrasing approaches: (i) BEAM, which adds to the provided reference the the $n$ best hypotheses in the beam of a baseline paraphraser, and (ii) SAMPLED, which samples from the top $80 \%$ of the probability mass at each time step (Edunov et al., 2018). For the sentence encoding methods, we also include (iii) RANDOM, where randomly selected cluster codes are used at training and test time.

As a topline, we compare against manually paraphrased references (HUMAN), which we produce for a subset of 500 sentences from the de-en test set. Two native English speakers together produced five paraphrases per reference (alternately two or three paraphrases). They were instructed to craft paraphrases that were maximally different (lexically and syntactically) from both the reference and the other paraphrases (to which they had access), without altering the original meaning.

\subsection{Paraphrase model training}

We train our paraphrasers using data from Parabank 2 (Hu et al., 2019b), containing $\approx 20 \mathrm{M}$ sentences with up to 5 paraphrases each, of which we use the first paraphrase only. We preprocess by removing duplicate sentences and those longer than 100 words and then segment into subwords using SentencePiece (Kudo and Richardson, 2018) (unigram model (Kudo, 2018) of size 16k). The data splits are created by randomly shuffling the data and reserving $3 \mathrm{k}$ pairs each for dev and test. For syntactic sentence encoding methods, we use the Berkeley Parser (Petrov et al., 2006) (internal tokenisation and prioritizing accuracy) and prune trees to a depth of 4 for $\approx 6 \mathrm{M}$ distinct trees. ${ }^{4}$

Paraphrase models are Transformer base models (Vaswani et al., 2017) (Cf. App. B for details). All models are trained using the Marian NMT toolkit (Junczys-Dowmunt et al., 2018), except for SAMPLED and the constraint approach, for which we use the Sockeye toolkit (Hieber et al., 2018), since Marian does not support these features.

For baseline models, we produce $n$ additional references by taking the $n$-best in the beam (using a beam size of 20 , which is the maximum number of additional references we test). For models using cluster codes, paraphrases are produced by selecting the $n$-best cluster codes at the first decoding step and then decoding each of these hypotheses using separate beam searches (of size 6).

\section{Paraphrase Adequacy and Diversity}

\subsection{Adequacy}

To ensure our automatically produced paraphrases are of sufficient quality, we first assess their adequacy (i.e., faithfulness to the original meaning). We determine adequacy by manually eval-

\footnotetext{
${ }^{4}$ Cf. App. A for the number of trees at different depths.
} 


\begin{tabular}{|c|c|c|c|}
\hline Reference & DA & What provoked Lindsay Lohan to such very strange actions is currently completely unclear. & Now they have come to an agreement. \\
\hline BEAM & 91.7 & $\begin{array}{l}\text { What caused Lindsay Lohan to do such strange things is not clear at the moment. } \\
\text { What provoked Lindsay Lohan's strange actions is not clear at the moment. } \\
\text { What has provoked Lindsay Lohan's strange actions is not clear at the moment. }\end{array}$ & $\begin{array}{l}\text { Now they've made a deal. } \\
\text { Now they've reached a deal. } \\
\text { Now they made a deal. }\end{array}$ \\
\hline SAMPLED & 85.0 & $\begin{array}{l}\text { What prompted Lindsay Lohan's most extraordinary actions? } \\
\text { What made Lindsay Lohan act so weird? } \\
\text { What inspired Lindsay Lohan to do such odd things? }\end{array}$ & $\begin{array}{l}\text { And now they've agreed. } \\
\text { And now they've agreed. } \\
\text { They've reached an agreement. }\end{array}$ \\
\hline LASER & 90.1 & $\begin{array}{l}\text { What provoked Lindsay Lohan to act so strangely is not clear at the moment. } \\
\text { It's not clear what provoked Lindsay Lohan to act so strangely. } \\
\text { It's not clear what prompted Lindsay Lohan to act so strangely. }\end{array}$ & $\begin{array}{l}\text { Now they've reached a deal. } \\
\text { Now they've agreed. } \\
\text { Now they've agreed }\end{array}$ \\
\hline TREELstM & 88.0 & $\begin{array}{l}\text { What provoked Lindsay Lohan to do such a strange thing is not clear at the moment. } \\
\text { It is not clear at this time what provoked Lindsay Lohan to do such strange things. } \\
\text { The reason that Lindsay Lohan has been provoked by these very strange actions is not clear } \\
\text { at the moment. }\end{array}$ & $\begin{array}{l}\text { Now they made a deal. } \\
\text { Now they've made a deal. } \\
\text { They've already made a deal. }\end{array}$ \\
\hline HUMAN & 95.2 & $\begin{array}{l}\text { It is currently totally unclear what made Lindsay Lohan do such strange things. } \\
\text { The cause of Lindsay Lohan's strange actions is really not clear at the moment. } \\
\text { The reasons behind Lindsay Lohan's such bizarre acts are completely obscure for now. }\end{array}$ & $\begin{array}{l}\text { They have now come to an agreement. } \\
\text { An agreement has now been made. } \\
\text { They have reached an agreement. }\end{array}$ \\
\hline
\end{tabular}

Table 1: Direct assessment (DA) adequacy scores for the BEAM and SAMPLED baseline, the two diverse approaches and human paraphrases for the 100-sentence de-en subset. We also provide each method's top 3 paraphrases for two references.

uating paraphrases of the first 100 sentences of the de-en test set. We compare a subset of the automatic methods (BEAM, SAMPLED, LASER, TREELSTM) as well as HUMAN. 5 annotators ( 2 native and 3 fluent English speakers) rated the paraphrases' adequacy using DA, indicating how well $(0-100)$ the official reference's meaning is preserved by its paraphrases. 25 judgments were collected per sentence (sampling from each system's top 5 paraphrases) System-level scores are produced by averaging across all annotations.

The results and examples of some of the paraphrased references are given in Tab. 1 (more examples are given in App. G). Whilst the task is inherently subjective, we see a clear preference for human paraphrases, providing a reference point for interpreting the scores. The automatic paraphrase systems are not far behind, and the scores are further corroborated by the lowest score being assigned to the sampled output, which we expect to be less faithful to the reference meaning.

\subsection{Diversity}

We evaluate the diversity of paraphrased references using two diversity scores (DS):

$$
\mathrm{DS}_{\mathrm{x}}=\frac{1}{|Y|(|Y|-1)} \sum_{y \in Y} \sum_{y^{\prime} \in Y, y^{\prime} \neq y} 1-\Delta_{\mathrm{x}}\left(y, y^{\prime}\right)
$$

where $Y$ is the set of paraphrases of a sentence produced by a given system, and $\Delta_{\mathrm{X}}$ calculates the similarity of paraphrases $y$ and $y^{\prime}$. We use two different functions: $\Delta_{B O W}$ (for lexical similarity) and $\Delta_{\text {tree }}$ (for syntactic similarity). Both give scores between 1 (identical) and 0 (maximally diverse),
$\mathbf{D S}_{\text {Bow }}$ is the lexical overlap between the sets of words in two paraphrases. $\Delta_{B O W}\left(y, y^{\prime}\right)$ corresponds to the number of unique words in common between $y$ and $y^{\prime}$, divided by their mean length.

DS $_{\text {tree }}$ uses $\Delta_{\text {tree }}$, the average tree kernel similarity score between paraphrases. We compute tree kernels using the "subset tree" (SST) comparison tree kernel similarity function presented in (Moschitti, 2006, §2.2), with a decay value of $\lambda=0.5$, and excluding leaves $(\sigma=0)$.

\begin{tabular}{|c|c|c|c|c|}
\hline$n$ & Method & $\mathrm{DS}_{B O W}$ & $\mathrm{DS}_{\text {tree }}$ & BLEU \\
\hline 0 & none & - & - & 29.8 \\
\hline \multirow{5}{*}{5} & RANDOM & 0.10 & 0.01 & 34.8 \\
\hline & BEAM & 0.22 & 0.30 & 37.0 \\
\hline & LASER & 0.24 & 0.33 & 37.5 \\
\hline & TREELSTM & 0.28 & 0.47 & 37.7 \\
\hline & SAMPLED & 0.41 & 0.56 & 40.1 \\
\hline \multirow{3}{*}{$5^{*}$} & SAMPLED & 0.40 & 0.55 & 47.0 \\
\hline & Constraints & 0.19 & 0.30 & 56.5 \\
\hline & HUMAN & 0.80 & 0.68 & 48.9 \\
\hline \multirow{5}{*}{20} & RANDOM & 0.10 & 0.01 & 34.8 \\
\hline & BEAM & 0.27 & 0.37 & 39.7 \\
\hline & LASER & 0.31 & 0.45 & 41.3 \\
\hline & TREELSTM & 0.32 & 0.53 & 41.0 \\
\hline & SAMPLED & 0.51 & 0.65 & 47.3 \\
\hline \multirow[t]{2}{*}{$\infty$} & Constraints & 0.21 & 0.28 & 46.4 \\
\hline & MT submissions & 0.37 & 0.51 & - \\
\hline
\end{tabular}

Table 2: Diversity scores (DS) of paraphrased references averaged over all into-English test sets, where $n$ is the number of paraphrases. The final row indicates diversity among MT outputs. * indicates results just for the 500 -sentence de-en subset. The final column is the average BLEU score.

The results (Tab. 2) show that all methods other than RANDOM give more diversity than BEAM. Shu et al.'s cluster code method generates diverse paraphrases. As expected, random cluster codes are not helpful, producing mostly identical para- 
phrases differing only in the cluster code. Diversity increases for all methods as paraphrases are added. TREELSTM produces structurally more diverse paraphrases than LASER and has high lexical diversity too, despite codes being entirely syntactic, suggesting that structural diversity leads to varied lexical choices. The most lexically and structurally diverse method (except for HUMAN), is in fact the strong baseline SAMPLED, which is likely due to the noise added with the method.

The increased diversity is generally reflected by an increase in the average BLEU score (final column of Tab. 2). These higher BLEU scores indicate that the additional paraphrases are better covering the translation space of the MT outputs, but it remains to be seen whether this concerns the space of valid and/or invalid translations. In contrast, some of the diversity makes less of an impact on the BLEU score; the gap in syntactic diversity between LASER and TREELSTM (+20 references) is not reflected in a similar gap in BLEU score, indicating that this added diversity is not relevant to the evaluation of these specific MT outputs.

\section{Metric Correlation Results}

The correlation results for each of the metrics (both system- and segment-level) for different numbers of additional references ${ }^{5}$ (aggregated full results) are shown in Tab. 3a and Tab. $3 b$ (for the de-en 500-sample subset). We aggregate the main results to make them easier to interpret by averaging over all into-English test sets (the Ave. column) and we also provide the gains for the language pairs that gave the smallest and greatest gains (Min and Max respectively). Full raw results can be found in App. D.

System-level Adding paraphrased references does not significantly hurt performance, and usually improves it; we see small gains for most languages (Ave. column), although the size of the gain varies, and correlations for two directions (fien and gu-en) are degraded but non-significantly (shown by the small negative minimum gains).

Fig. 1 (top) shows that for the diverse approaches, the average gain is positively correlated with the method's diversity: increased diversity does improve coverage of the valid translation space. This positive correlation holds for all directions for which adding paraphrases helps

\footnotetext{
${ }^{5}$ The table only reports up to 5 paraphrases; adding 10 or 20 did not improve any of the correlations further.
}

(i.e., all except fi-en and gu-en). For these exceptions, none of the methods significantly improves over the baseline, and RANDOM gives as good if not marginally better results. The constraints approach achieves the highest average gain, suggesting that it is more efficiently targeting the space of valid translations, even though its paraphrases are significantly less diverse (Tab. 2).

Finally, and in spite of these improvements, we note that all systems fall far short of the best WMT19 metrics, shown in the last row. Automatic paraphrases do not seem to address the weakness of BLEU as an automatic metric.

Segment-level Similar results can be seen at the segment level, with most diverse approaches showing improvements over the baseline (this time SENTBLEU) and a minority showing nonsignificant deteriorations (i.e., no change). The diversity of the approaches is again positively correlated with the gains seen (Fig. 1, bottom), with the exception of zh-en, for no easily discernable reason.

The best result of the diverse approaches is again achieved by the SAMPLED baseline.

The constraint-based approach achieves good scores, comparable to SAMPLED, despite an anomalously poor score for one language pair (for kk-en, with a degradation of 0.097. This approach also had the highest BLEU scores, however, suggesting that the targeted paraphrasing approach here missed its mark.

De-en 500-sentence subset The general pattern shows the same as the averages over all languages in Tab. 3a, with the more diverse methods (especially SAMPLED) resulting in the greatest gains. The human results also follow this pattern, resulting in the highest gains of all at the system level. Interestingly, the constrained system yields higher average BLEU scores than HUMAN (Tab. 2) yet a comparable system correlation gain, indicating it targets more of the invalid translation space. For this particular subset, the constraints-based approach helps slightly more at the segment level than the system level, even surpassing the human paraphrases in terms of relative gains, despite it having remarkably less diversity.

\section{Discussion}

Does diversity help? In situations where adding paraphrases helps (which is the case for a majority 


\begin{tabular}{|c|c|c|c|c|c|c|c|c|c|}
\hline \multirow{5}{*}{$\begin{array}{l}\text { Approach } \\
\begin{array}{l}\text { Baselines } \\
(+5)\end{array}\end{array}$} & \multirow{5}{*}{$\begin{array}{l}\text { Method } \\
\text { BEAM } \\
\text { RANDOM } \\
\text { SAMPLED }\end{array}$} & \multicolumn{3}{|c|}{ System Gains } & \multicolumn{3}{|c|}{ Segment Gains } & \multirow{2}{*}{\multicolumn{2}{|c|}{$\begin{array}{c}\text { System Segment } \\
\text { de-en }\end{array}$}} \\
\hline & & \multirow{2}{*}{$\begin{array}{r}\text { Ave. } \\
0.020\end{array}$} & \multirow{2}{*}{$\begin{array}{r}\text { Min } \\
-0.006\end{array}$} & \multirow{2}{*}{$\begin{array}{r}\text { Max } \\
0.059\end{array}$} & \multirow{2}{*}{$\begin{array}{c}\text { Ave. } \\
0.013\end{array}$} & \multirow{2}{*}{$\begin{array}{r}\text { Min } \\
-0.001\end{array}$} & \multirow{2}{*}{$\frac{\text { Max }}{0.029}$} & & \\
\hline & & & & & & & & 0.040 & 0.021 \\
\hline & & 0.017 & 0.000 & 0.046 & 0.007 & -0.002 & 0.017 & 0.031 & 0.017 \\
\hline & & 0.024 & -0.002 & 0.067 & 0.017 & -0.004 & 0.044 & 0.044 & 0.043 \\
\hline & LASER & 0.017 & -0.000 & 0.048 & 0.009 & -0.003 & 0.025 & 0.034 & 0.022 \\
\hline Diversity $(+1)$ & TreeLstm & 0.017 & -0.000 & 0.048 & 0.011 & -0.002 & 0.027 & 0.031 & 0.011 \\
\hline & LASER & 0.020 & -0.004 & 0.056 & 0.011 & -0.002 & 0.033 & 0.040 & 0.022 \\
\hline Divers1 & TreeLstm & 0.020 & -0.004 & 0.057 & 0.013 & -0.004 & 0.030 & 0.044 & 0.008 \\
\hline Output- & LASER & 0.012 & -0.006 & 0.041 & 0.006 & -0.001 & 0.016 & 0.032 & 0.015 \\
\hline specific $(+1)$ & TreELstM & 0.014 & -0.007 & 0.041 & 0.007 & -0.005 & 0.016 & 0.039 & 0.011 \\
\hline Constraints & 4-grams & 0.025 & -0.002 & 0.061 & 0.002 & -0.097 & 0.072 & -0.027 & 0.035 \\
\hline Hum & & & & - & - & - & - & 0.039 & 0.037 \\
\hline WMT-19 best & Multiple & 0.079 & 0.010 & 0.194 & 0.117 & 0.072 & 0.145 & - & \\
\hline
\end{tabular}

(a) Average and minimum and maximum gains over all into-English test sets

(b) 500-sample subset

Table 3: Absolute gains in correlation (with respect to the true BLEU and sentenceBLEU baseline correlations). Significant gains (except for averages) are marked in bold ( $p \leq 0.05$ ). Full results per language pair are provided in App. D. WMT-19 best refers to the best metric scores from the official shared task (the best metric can be different for each language pair).

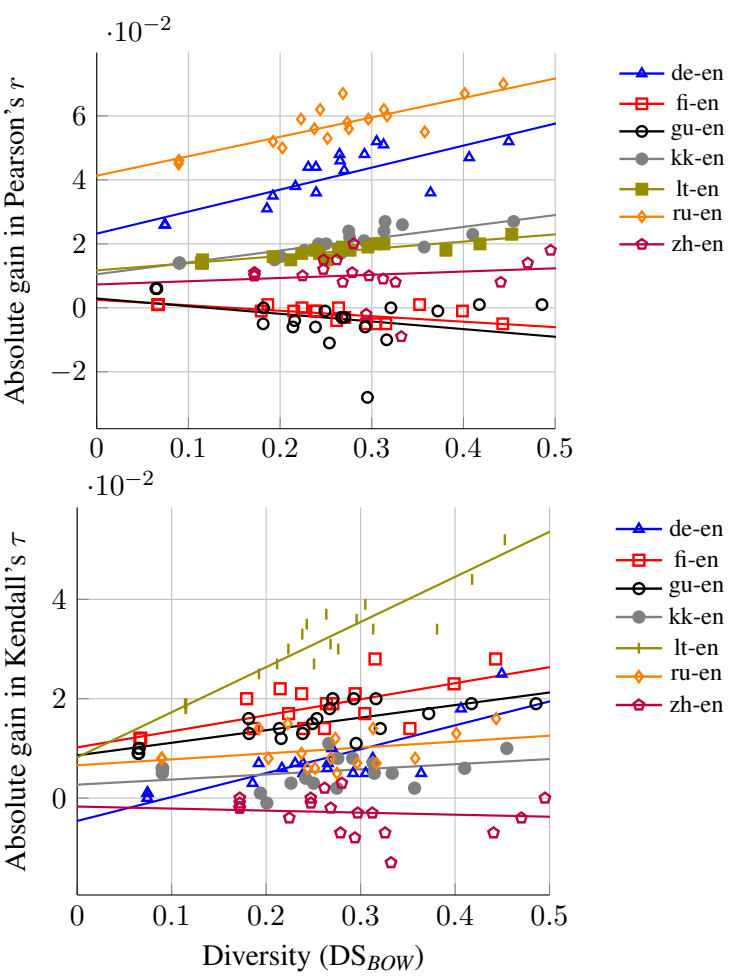

Figure 1: Lexical diversity versus absolute correlation gain at the system level (top) and segment level (bottom) for a variety of paraphrase systems $(+2,+5,+10$ and +20 references $)$.

of language directions), the diversity of those paraphrases tends to positively correlate with gains in metric performance for both BLEU and SENTBLEU. The adequacy of the paraphrases appears to be a less important factor, shown by the fact that the best automatic diverse method at both levels was the SAMPLED baseline, the most diverse but the least adequate. ${ }^{6}$ The comparison against human paraphrases on the de-en subsample suggests room for improvement in automated techniques, at least at the system level, where all automatic metrics are beaten by HUMAN paraphrases, which are both more diverse and more adequate.

However, diversity is not everything; although HUMAN has nearly twice the lexical diversity of SAMPLED, it improves BLEU only somewhat and harms sentence BLEU. On the other side, targeted constraints have relatively low diversity, but higher correlation gains. Diversity itself does not necessarily result in coverage of the space occupied by good translation hypotheses.

What effect do more references have? Diversity increases the more paraphrases there are and it is positively correlated with gains for most language directions. However, improvements are slight, especially with respect to what we would hope to achieve (using human references results in much more diversity and also greater improvements). The relationship between the number of extra references and system-level correlations shown in Fig. 2 suggests that increasing the number of references results in gains, but for most test sets, the initial paraphrase has the most impact and the subsequent ones lead to lesser gains or even occasional deteriorations. Similar results are seen at the segment level.

\footnotetext{
${ }^{6}$ We did not categorize our adequacy judgments, but SAMPLED's lower adequacy could be caused by (the relatively harmless) deletion of information (anecdotally supported in Tab. 1).
} 


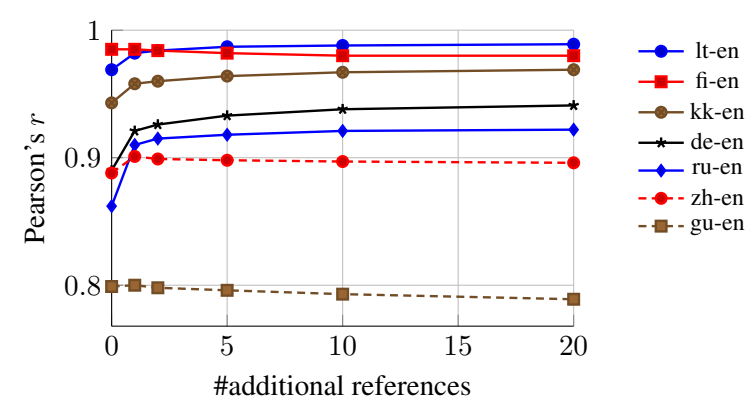

Figure 2: TREeLstM system-level correlations (+0-20).

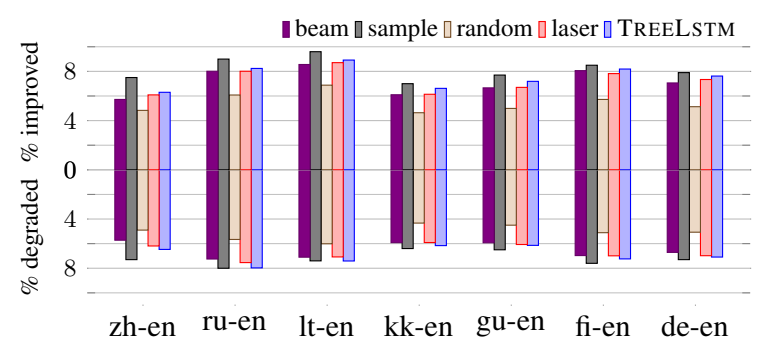

Figure 3: \% improved and degraded (with respect to singlereference sentence-BLEU) for methods with +5 references.

Why are gains only slight? With respect to the SENTBLEU baseline, we calculate the percentage of comparisons for which the decision is improved (the baseline scored the worse translation higher than the better one and the new paraphraseaugmented metric reversed this) ${ }^{7}$ and for which the decision is degraded (opposite reversal). The results (Fig. 3) show that although all the systems improve a fair number of comparisons (up to $9.6 \%$ ), they degrade almost as many. So, while paraphrasing adds references that represent the space of valid translations, references are indeed being added that match with the space of invalid ones too. Interestingly, the same pattern can be seen for human paraphrases, $6.46 \%$ of comparisons being degraded vs. $8.30 \%$ improved, suggesting that even when gold standard paraphrases are produced, the way in which the references are used by SENTBLEU still rewards some invalid translations, though the balance is shifted slightly in favour of valid translations. This suggests that at least at the segment level, BLEU is a balancing act between rewarding valid translations and avoiding rewarding invalid ones. Some of these effects may be smoothed out in system-level BLEU but there is still likely to be an effect. It is worth noting that for the two languages directions, fi-en and gu-en, for which diversity was negatively cor-

\footnotetext{
${ }^{7}$ 'Better' and 'worse' systems are determined by the official DA human assessments of MT quality.
}

related with correlation gain (i.e., diversity could be harming performance), the most conservative approach (RANDOM) leads to some of the best results.

What is the effect on individual $n$-grams? We study which new $n$-grams are being matched by the additional references for the two language directions with the largest system-level correlation gain (ru-en and de-en). For each sentence, we collect and count the $n$-grams that were not in the original reference but where in the five paraphrased references of BEAM (missing $n$-grams), ${ }^{8}$ accumulated across all test set sentences. We also looked at the most frequent $n$-grams not found at all, even with the help of the paraphrases (i.e., the unrewarded $n$-grams from Sec. 3.2). The results are in Table 4.

Unsurprisingly, most 1-grams are common grammatical words (e.g., $a$, of, to, in, the) that may be present (or not) in any sentence; it is hard to draw any conclusions. For 4-grams, however, we see some interesting patterns. Present in both lists are acronym variants such as $U . S$. for 'United States' and $p . m$. for 'afternoon' or the 24-hour clock; their presence on both sides indicates success in sometimes grabbing this variant as well as failure to do so consistently. We also see phrasal variants such as, according to and, " he said. These last points corroborate a point made by Freitag et al. $(2020, \S 7.2)$ that references may omit these common variants. It also suggests a more focused method for generating paraphrases: identify a high-precision set of common variants, and ensure their presence in the set of references, via constrained decoding or other means (in the spirit of Meteor's (Denkowski and Lavie, 2011) synonym-based matching). We note however, that our paraphrasing methods do seem to contain complementary information as they also tend to improve Meteor too (see results in App. F).

\section{Conclusion}

We studied the feasibility of using diverse automatic paraphrasing of English references to improve BLEU. Although increased diversity of paraphrases does lead to increased gains in correlation with human judgments at both the system and segment levels, the gains are small and inconsistent. We can do a slightly better job by using

\footnotetext{
${ }^{8}$ Using sacreBLEU's default v13a tokenization.
} 


\begin{tabular}{|c|c|c|}
\hline $\mathrm{N}$ & newly matched ngrams & missing ngrams \\
\hline 1 & $\begin{array}{l}\text { a (494) of }(480),(442) \text { to }(370) \text { in }(364) \text { The }(315) \text { the }(273) \\
\text { is }(204) \text { for }(196) \text { has }(196) \text { on }(193) \text { was }(179) \text { have }(171) \\
\text { that }(166) \text { be }(155) \text { at }(145) \text { been }(140) \text { with }(138) \text { and }(134)\end{array}$ & $\begin{array}{l}\text { to }(921) \text { in }(921) \text { on }(870) \text { is }(802) \text { of }(798) \text { a }(786) \text { for }(568) \\
\text { The }(556) \text { with }(509) \text { it }(508) \text { has }(505) \text { are }(482) \text { by }(480) \\
\text { was }(478) \text { have }(449) \text { - }(443) \text { at }(437) \text { as }(426) \text { which }(386)\end{array}$ \\
\hline 4 & $\begin{array}{l}\text { U.S. . (63) the U.S (39), as well as (19) p. m. (15) for the } \\
\text { first time (13) in accordance with the (12) the United States } \\
\text {, (11) in the United States }(10) \text { a member of the }(10) \text { of the } \\
\text { United States (9) The U.S (9). m . on (9), in order to (9) } \\
\text { the United States and (8), of course, (8). S . Navy (8) . } \\
\text { m., (8) the Chinese Academy of (8) Chinese Academy of } \\
\text { Engineering (8) the renaming of the (7) }\end{array}$ & $\begin{array}{l}\text { U.S . (136), according to the (99), " he said (77) the U.S } \\
\text { (55) of the United States ( } 48 \text { ) of the Ministry of (39) the end } \\
\text { of the (38)," said the (37) same time, the (36), such as the } \\
\text { (36) as well as the (35) (Xinhua) - (34) and so on . (33), } \\
\text { he said. (32) the head of the (32), the head of (31), as well } \\
\text { as (30) on the basis of (30), and so on (29) }\end{array}$ \\
\hline
\end{tabular}

Table 4: Most frequently newly matched and missing $n$-grams for the de-en and ru-en test sets for BEAM $(+5)$.

cues from the system outputs themselves to produce paraphrases providing a helpful form of "targeted" diversity. The comparison with manually produced paraphrases shows that there is room for improvement, both in terms of how much diversity is achieved and how much BLEU can be improved. However, the lack of any improvement in some languages points to how hard it is to target this "right kind" of diversity a priori; this, together with the relatively limited gains overall (especially in comparison with the best WMT19 metrics), suggests an intrinsic limit to BLEU's capacity to handle multiple references.

\section{Acknowledgements}

We would like to thank MT Marathon 2019, during which this work was initiated and which also provide us with initial computing resources. This work was supported by funding from the European Union's Horizon 2020 research and innovation programme under grant agreements No 825299 (GoURMET), 825303 and the UK Engineering and Physical Sciences Research Council (EPSRC) fellowship grant EP/S001271/1 (MTStretch).

\section{References}

Joshua Albrecht and Rebecca Hwa. 2008. The role of pseudo references in MT evaluation. In Proceedings of the Third Workshop on Statistical Machine Translation, pages 187-190, Columbus, Ohio. Association for Computational Linguistics.

Marianna Apidianaki, Guillaume Wisniewski, Anne Cocos, and Chris Callison-Burch. 2018. Automated paraphrase lattice creation for HyTER machine translation evaluation. In Proceedings of the 2018 Conference of the North American Chapter of the Association for Computational Linguistics: $\mathrm{Hu}$ man Language Technologies, Volume 2 (Short Papers), pages 480-485, New Orleans, Louisiana. Association for Computational Linguistics.
Mikel Artetxe and Holger Schwenk. 2019. Massively multilingual sentence embeddings for zeroshot cross-lingual transfer and beyond. Transactions of the Association for Computational Linguistics, 7:597-610.

Satanjeev Banerjee and Alon Lavie. 2005. METEOR: An automatic metric for MT evaluation with improved correlation with human judgments. In Proceedings of the ACL Workshop on Intrinsic and Extrinsic Evaluation Measures for Machine Translation and/or Summarization, pages 65-72, Ann Arbor, Michigan. Association for Computational Linguistics.

Loïc Barrault, Ondřej Bojar, Marta R. Costa-jussà, Christian Federmann, Mark Fishel, Yvette Graham, Barry Haddow, Matthias Huck, Philipp Koehn, Shervin Malmasi, Christof Monz, Mathias Müller, Santanu Pal, Matt Post, and Marcos Zampieri. 2019. Findings of the 2019 conference on machine translation (WMT19). In Proceedings of the Fourth Conference on Machine Translation (Volume 2: Shared Task Papers, Day 1), pages 1-61, Florence, Italy. Association for Computational Linguistics.

Chris Callison-Burch, Miles Osborne, and Philipp Koehn. 2006. Re-evaluating the role of Bleu in machine translation research. In 11th Conference of the European Chapter of the Association for Computational Linguistics, Trento, Italy. Association for Computational Linguistics.

Emilie Colin and Claire Gardent. 2018. Generating syntactic paraphrases. In Proceedings of the 2018 Conference on Empirical Methods in Natural Language Processing, pages 937-943, Brussels, Belgium. Association for Computational Linguistics.

Michael Denkowski and Alon Lavie. 2011. Meteor 1.3: Automatic metric for reliable optimization and evaluation of machine translation systems. In Proceedings of the Sixth Workshop on Statistical Machine Translation, pages 85-91, Edinburgh, Scotland. Association for Computational Linguistics.

Michael Denkowski and Alon Lavie. 2014. Meteor universal: Language specific translation evaluation for any target language. In Proceedings of the EACL 2014 Workshop on Statistical Machine Translation. 
Markus Dreyer and Daniel Marcu. 2012. HyTER: Meaning-equivalent semantics for translation evaluation. In Proceedings of the 2012 Conference of the North American Chapter of the Association for Computational Linguistics: Human Language Technologies, pages 162-171, Montréal, Canada. Association for Computational Linguistics.

Sergey Edunov, Myle Ott, Michael Auli, and David Grangier. 2018. Understanding back-translation at scale. In Proceedings of the 2018 Conference on Empirical Methods in Natural Language Processing, pages 489-500, Brussels, Belgium. Association for Computational Linguistics.

Christian Federmann, Oussama Elachqar, and Chris Quirk. 2019. Multilingual whispers: Generating paraphrases with translation. In Proceedings of the 5th Workshop on Noisy User-generated Text (WNUT 2019), pages 17-26, Hong Kong, China. Association for Computational Linguistics.

Markus Freitag, David Grangier, and Isaac Caswell. 2020. BLEU might be Guilty but References are not Innocent. arXiv.

Yvette Graham, Timothy Baldwin, Alistair Moffat, and Justin Zobel. 2013. Continuous measurement scales in human evaluation of machine translation. In Proceedings of the 7th Linguistic Annotation Workshop and Interoperability with Discourse, pages 33-41, Sofia, Bulgaria. Association for Computational Linguistics.

Felix Hieber, Tobias Domhan, Michael Denkowski, David Vilar, Artem Sokolov, Ann Clifton, and Matt Post. 2018. The sockeye neural machine translation toolkit at AMTA 2018. In Proceedings of the 13th Conference of the Association for Machine Translation in the Americas (Volume 1: Research Papers), pages 200-207, Boston, MA. Association for Machine Translation in the Americas.

J. Edward Hu, Huda Khayrallah, Ryan Culkin, Patrick Xia, Tongfei Chen, Matt Post, and Benjamin Van Durme. 2019a. Improved lexically constrained decoding for translation and monolingual rewriting. In Proceedings of the 2019 Conference of the North American Chapter of the Association for Computational Linguistics: Human Language Technologies, Volume 1 (Long and Short Papers), pages 839-850, Minneapolis, Minnesota. Association for Computational Linguistics.

J. Edward Hu, Abhinav Singh, Nils Holzenberger, Matt Post, and Benjamin Van Durme. 2019b. Largescale, diverse, paraphrastic bitexts via sampling and clustering. In Proceedings of the 23rd Conference on Computational Natural Language Learning (CoNLL), pages 44-54, Hong Kong, China. Association for Computational Linguistics.

Mohit Iyyer, John Wieting, Kevin Gimpel, and Luke Zettlemoyer. 2018. Adversarial example generation with syntactically controlled paraphrase networks.
In Proceedings of the 2018 Conference of the North American Chapter of the Association for Computational Linguistics: Human Language Technologies, Volume 1 (Long Papers), pages 1875-1885, New Orleans, Louisiana. Association for Computational Linguistics.

Marcin Junczys-Dowmunt, Kenneth Heafield, Hieu Hoang, Roman Grundkiewicz, and Anthony Aue. 2018. Marian: Cost-effective high-quality neural machine translation in $\mathrm{C}++$. In Proceedings of the 2nd Workshop on Neural Machine Translation and Generation, pages 129-135, Melbourne, Australia. Association for Computational Linguistics.

David Kauchak and Regina Barzilay. 2006. Paraphrasing for automatic evaluation. In Proceedings of the Human Language Technology Conference of the NAACL, Main Conference, pages 455-462, New York City, USA. Association for Computational Linguistics.

Taku Kudo. 2018. Subword regularization: Improving neural network translation models with multiple subword candidates. In Proceedings of the 56th Annual Meeting of the Association for Computational Linguistics (Volume 1: Long Papers), pages 6675, Melbourne, Australia. Association for Computational Linguistics.

Taku Kudo and John Richardson. 2018. SentencePiece: A simple and language independent subword tokenizer and detokenizer for neural text processing. In Proceedings of the 2018 Conference on Empirical Methods in Natural Language Processing: System Demonstrations, pages 66-71, Brussels, Belgium. Association for Computational Linguistics.

Qingsong Ma, Johnny Wei, Ondřej Bojar, and Yvette Graham. 2019. Results of the WMT19 metrics shared task: Segment-level and strong MT systems pose big challenges. In Proceedings of the Fourth Conference on Machine Translation (Volume 2: Shared Task Papers, Day 1), pages 62-90, Florence, Italy. Association for Computational Linguistics.

Nitin Madnani, Necip Fazil Ayan, Philip Resnik, and Bonnie Dorr. 2007. Using paraphrases for parameter tuning in statistical machine translation. In Proceedings of the Second Workshop on Statistical Machine Translation, pages 120-127, Prague, Czech Republic. Association for Computational Linguistics.

Jonathan Mallinson, Rico Sennrich, and Mirella Lapata. 2017. Paraphrasing revisited with neural machine translation. In Proceedings of the 15th Conference of the European Chapter of the Association for Computational Linguistics: Volume 1, Long Papers, pages 881-893, Valencia, Spain. Association for Computational Linguistics.

Nitika Mathur, Timothy Baldwin, and Trevor Cohn. 2020. Tangled up in BLEU: Reevaluating the evaluation of automatic machine translation evaluation 
metrics. In Proceedings of the 58th Annual Meeting of the Association for Computational Linguistics, pages 4984-4997, Online. Association for Computational Linguistics.

Alessandro Moschitti. 2006. Making tree kernels practical for natural language learning. In 11th Conference of the European Chapter of the Association for Computational Linguistics, Trento, Italy. Association for Computational Linguistics.

Courtney Napoles, Chris Callison-Burch, and Matt Post. 2016. Sentential paraphrasing as black-box machine translation. In Proceedings of the 2016 Conference of the North American Chapter of the Association for Computational Linguistics: Demonstrations, pages 62-66, San Diego, California. Association for Computational Linguistics.

Kishore Papineni, Salim Roukos, Todd Ward, and WeiJing Zhu. 2002. Bleu: a method for automatic evaluation of machine translation. In Proceedings of the 40th Annual Meeting of the Association for Computational Linguistics, pages 311-318, Philadelphia, Pennsylvania, USA. Association for Computational Linguistics.

Slav Petrov, Leon Barrett, Romain Thibaux, and Dan Klein. 2006. Learning accurate, compact, and interpretable tree annotation. In Proceedings of the 21st International Conference on Computational Linguistics and 44th Annual Meeting of the Association for Computational Linguistics, pages 433-440, Sydney, Australia. Association for Computational Linguistics.

Matt Post. 2018. A call for clarity in reporting BLEU scores. In Proceedings of the Third Conference on Machine Translation: Research Papers, pages 186191, Brussels, Belgium. Association for Computational Linguistics.

Matt Post and David Vilar. 2018. Fast lexically constrained decoding with dynamic beam allocation for neural machine translation. In Proceedings of the 2018 Conference of the North American Chapter of the Association for Computational Linguistics: $\mathrm{Hu}$ man Language Technologies, Volume 1 (Long Papers), pages 1314-1324, New Orleans, Louisiana. Association for Computational Linguistics.

Aaditya Prakash, Sadid A. Hasan, Kathy Lee, Vivek Datla, Ashequl Qadir, Joey Liu, and Oladimeji Farri. 2016. Neural paraphrase generation with stacked residual LSTM networks. In Proceedings of COLING 2016, the 26th International Conference on Computational Linguistics: Technical Papers, pages 2923-2934, Osaka, Japan. The COLING 2016 Organizing Committee.

Raphael Shu, Hideki Nakayama, and Kyunghyun Cho. 2019. Generating diverse translations with sentence codes. In Proceedings of the 57th Annual Meeting of the Association for Computational Linguistics, pages 1823-1827, Florence, Italy. Association for Computational Linguistics.
Kai Sheng Tai, Richard Socher, and Christopher D. Manning. 2015. Improved semantic representations from tree-structured long short-term memory networks. In Proceedings of the 53rd Annual Meeting of the Association for Computational Linguistics and the 7th International Joint Conference on Natural Language Processing (Volume 1: Long Papers), pages 1556-1566, Beijing, China. Association for Computational Linguistics.

Ashish Vaswani, Noam Shazeer, Niki Parmar, Jakob Uszkoreit, Llion Jones, Aidan N Gomez, Łukasz Kaiser, and Illia Polosukhin. 2017. Attention is all you need. In I. Guyon, U. V. Luxburg, S. Bengio, H. Wallach, R. Fergus, S. Vishwanathan, and R. Garnett, editors, Advances in Neural Information Processing Systems 30, pages 5998-6008. Curran Associates, Inc.

John Wieting and Kevin Gimpel. 2018. ParaNMT50M: Pushing the limits of paraphrastic sentence embeddings with millions of machine translations. In Proceedings of the 56th Annual Meeting of the Association for Computational Linguistics (Volume 1: Long Papers), pages 451-462, Melbourne, Australia. Association for Computational Linguistics.

Evan James Williams. 1959. Regression Analysis. Wiley, New York.

Qiongkai Xu, Juyan Zhang, Lizhen Qu, Lexing Xie, and Richard Nock. 2018. D-PAGE: diverse paraphrase generation. CoRR, abs/1808.04364.

Ryoma Yoshimura, Hiroki Shimanaka, Yukio Matsumura, Hayahide Yamagishi, and Mamoru Komachi. 2019. Filtering pseudo-references by paraphrasing for automatic evaluation of machine translation. In Proceedings of the Fourth Conference on Machine Translation (Volume 2: Shared Task Papers, Day 1), pages 521-525, Florence, Italy. Association for Computational Linguistics.

Liang Zhou, Chin-Yew Lin, and Eduard Hovy. 2006. Re-evaluating machine translation results with paraphrase support. In Proceedings of the 2006 Conference on Empirical Methods in Natural Language Processing, pages 77-84, Sydney, Australia. Association for Computational Linguistics. 


\section{A Number of distinct parse trees at different depths}

Table 5 shows the number of distinct pruned tree at different depths. We choose a depth of 4 for the syntactic sentence encoding methods in our experiments.

\begin{tabular}{rrrr}
\hline depth & no leaves & type/token & with leaves \\
\hline 1 & 16 & $0 \%$ & 16 \\
2 & 207,794 & $1.0 \%$ & 207,794 \\
3 & $2,158,114$ & $11.2 \%$ & $2,629,907$ \\
4 & $6,089,874$ & $31.6 \%$ & $10,631,249$ \\
5 & $8,865,720$ & $46.1 \%$ & $14,102,645$ \\
\hline$\infty$ & $13,054,272$ & $68.1 \%$ & $17,362,448$ \\
\hline
\end{tabular}

Table 5: Number of distinct pruned trees in different depths with and without leaves in the parsed data.

\section{B Paraphraser training details}

All paraphrase models are Transformer base models (Vaswani et al., 2017): 6 layers, 8 heads, word embedding dimension of 512, feedforward dimension of 2048. We set dropout to 0.1 and tie all embeddings to the output layer with a shared vocabulary size of 33,152 . We use the same vocabulary (including the 256 cluster codes) for all models. We adopt Adam optimisation with a scheduled learning rate (initial $3 \times 10^{-4}$ ) and mini-batch size of 64. We train each model on 4 GTX Titan X GPUs with a gradient update delay of 2, and select the final model based on validation BLEU.

\section{Sentence clustering training details}

We set $k$ to 256 for $k$-means clustering. We train TREeLstm sentence encoders using Adagrad with a learning rate of 0.025 , weight decay of $10^{-4}$ and batch size of 400 for a maximum of 20 iterations. We set the model size to 256 and limit the maximum number of child nodes to 10 .

\section{Full raw WMT19 results}

Table 7 shows the raw correlations of each each paraphrase-augmented BLEU metric on WMT19 (system-level results top and segment-level results bottom). These correspond to the raw scores used to calculate the gains of each method with respect to the true baseline (BLEU or sentenceBLEU) shown in the main results section in Table 3 . We indicate the best system from WMT19 as a point of reference.

\section{E Raw results for the de-en 500-sentence subset}

\begin{tabular}{llll}
\hline & & \multicolumn{2}{c}{ Correlation } \\
& Method & System & Segment \\
\hline Baseline & (sentence)BLEU & 0.895 & 0.026 \\
\hline \multirow{2}{*}{$\begin{array}{l}\text { Baselines } \\
(+5)\end{array}$} & BEAM & $\mathbf{0 . 9 3 4}$ & 0.048 \\
& RANDOM & $\mathbf{0 . 9 2 6}$ & 0.043 \\
SAMersity & LASER & $\mathbf{0 . 9 2 9}$ & 0.048 \\
$(+1)$ & TREELSTM & $\mathbf{0 . 9 2 6}$ & 0.037 \\
\hline Diversity & LASER & $\mathbf{0 . 9 3 5}$ & 0.049 \\
$(+5)$ & TREELSTM & $\mathbf{0 . 9 3 9}$ & 0.034 \\
\hline \hline Constraints & 4-gram & $\mathbf{0 . 9 3 3}$ & 0.064 \\
\hline Human & & $\mathbf{0 . 9 4 8}$ & 0.063 \\
\hline
\end{tabular}

Table 6: Correlations on the 500-sentence subset.

\section{F Results with the Meteor metric}

Although we focus on ways of improving BLEU using paraphrases in this article, as BLEU is the dominant metric, it is also interesting to look at how adding paraphrases could help similar metrics. We apply the same method to improving the Meteor metric (version 1.5) (Denkowski and Lavie, 2014), a metric which already integrates synonym support.

Summarised results (as gains with respect to the single-reference Meteor metric) are shown in Tab. 8 and raw results are shown in Tab. 9 for both system-level and segment-level correlations. We observe that the true baselines (Meteor and sentenceMeteor) are improved in both cases, possibly more so than BLEU and in different ways, showing that the information added by the paraphrases is complementary to the synonym support offered by Meteor.

\section{G Further examples of automatically paraphrased references}

We provide additional examples of paraphrased references. As can be seen from Table 10, TREELSTM gives us more diverse sentences compared to LASER. 


\begin{tabular}{|c|c|c|c|c|c|c|c|c|c|}
\hline Approach & Method & $\begin{array}{l}\text { de-en } \\
(16)\end{array}$ & $\begin{array}{l}\text { fi-en } \\
\text { (12) }\end{array}$ & $\begin{array}{l}\text { gu-en } \\
\text { (12) }\end{array}$ & $\begin{array}{l}\text { kk-en } \\
\text { (11) }\end{array}$ & $\begin{array}{l}\text { lt-en } \\
\text { (11) }\end{array}$ & $\begin{array}{l}\text { ru-en } \\
(14)\end{array}$ & $\begin{array}{l}\text { zh-en } \\
\text { (15) }\end{array}$ & Ave \\
\hline Baseline & BLEU & 0.890 & 0.985 & 0.799 & 0.943 & 0.969 & 0.862 & 0.888 & 0.905 \\
\hline \multirow{3}{*}{$\begin{array}{l}\text { Paraphrase } \\
\text { baselines } \\
(+5)\end{array}$} & BEAM & 0.928 & 0.984 & 0.793 & 0.961 & 0.986 & 0.921 & 0.900 & 0.925 \\
\hline & RANDOM & 0.916 & 0.986 & 0.805 & 0.957 & 0.983 & 0.908 & 0.898 & 0.922 \\
\hline & SAMPLED & 0.937 & 0.984 & 0.798 & 0.966 & 0.989 & 0.929 & 0.902 & 0.929 \\
\hline \multirow{2}{*}{$\begin{array}{l}\text { Diversity } \\
(+1)\end{array}$} & LASER & 0.919 & 0.987 & 0.799 & 0.957 & 0.981 & 0.909 & 0.904 & 0.922 \\
\hline & TreeLstm & 0.921 & 0.985 & 0.800 & 0.958 & 0.982 & 0.910 & 0.901 & 0.922 \\
\hline \multirow{2}{*}{$\begin{array}{l}\text { Diversity } \\
(+5)\end{array}$} & LASER & 0.934 & 0.985 & 0.795 & 0.963 & 0.987 & 0.918 & 0.896 & 0.925 \\
\hline & TreeLstm & 0.933 & 0.982 & 0.796 & 0.964 & 0.987 & 0.918 & 0.898 & 0.925 \\
\hline \multirow[t]{2}{*}{ Constraints } & 4-grams & 0.922 & 0.983 & 0.809 & 0.963 & 0.989 & 0.924 & 0.921 & 0.930 \\
\hline & WMT-19 best & $\begin{array}{l}\mathbf{0 . 9 5 0} * * \\
\text { (YISI-1_SRL) }\end{array}$ & $\begin{array}{l}\mathbf{0 . 9 9 5} \\
\text { (METEOR) }\end{array}$ & $\begin{array}{l}\mathbf{0 . 9 9 3} * * * \\
(\text { YISI-0) }\end{array}$ & $\begin{array}{l}\mathbf{0 . 9 9 8}^{*} * * \\
\text { (WMDO) }\end{array}$ & $\begin{array}{l}\text { 0.989* } \\
\text { (ESIM) }\end{array}$ & $\begin{array}{l}\mathbf{0 . 9 7 9} * * \\
\left(\mathrm{YISI}^{2}-1\right)\end{array}$ & $\begin{array}{l}\mathbf{0 . 9 8 8}^{* * * *} \\
\text { (ESIM) }\end{array}$ & 0.985 \\
\hline
\end{tabular}

(a) Pearson correlations at the system level.

\begin{tabular}{|c|c|c|c|c|c|c|c|c|c|}
\hline Approach & Method & $\begin{array}{l}\text { de-en } \\
(32000)\end{array}$ & $\begin{array}{l}\text { fi-en } \\
\text { (23952) }\end{array}$ & $\begin{array}{l}\text { gu-en } \\
(12192)\end{array}$ & $\begin{array}{l}\text { kk-en } \\
(11000)\end{array}$ & $\begin{array}{l}\text { lt-en } \\
(11000)\end{array}$ & $\begin{array}{l}\text { ru-en } \\
(28000)\end{array}$ & $\begin{array}{l}\text { zh-en } \\
(30000)\end{array}$ & Ave \\
\hline Baseline & sentenceBLEU & 0.055 & 0.228 & 0.175 & 0.368 & 0.251 & 0.114 & 0.317 & 0.215 \\
\hline \multirow{3}{*}{$\begin{array}{l}\text { Paraphrase } \\
\text { baselines } \\
(+5)\end{array}$} & BEAM & 0.061 & 0.250 & 0.189 & 0.371 & 0.281 & 0.129 & 0.317 & 0.228 \\
\hline & RANDOM & 0.056 & 0.240 & 0.184 & 0.374 & 0.269 & 0.122 & 0.315 & 0.223 \\
\hline & SAMPLED & 0.073 & 0.251 & 0.192 & 0.374 & 0.295 & 0.127 & 0.313 & 0.232 \\
\hline \multirow{2}{*}{$\begin{array}{l}\text { Diversity } \\
(+1)\end{array}$} & LASER & 0.061 & 0.244 & 0.187 & 0.368 & 0.276 & 0.121 & 0.314 & 0.225 \\
\hline & TREeLstM & 0.061 & 0.242 & 0.185 & 0.383 & 0.278 & 0.123 & 0.315 & 0.227 \\
\hline \multirow{2}{*}{$\begin{array}{l}\text { Diversity } \\
(+5)\end{array}$} & LASER & 0.062 & 0.245 & 0.187 & 0.372 & 0.284 & 0.123 & 0.315 & 0.227 \\
\hline & TREeLstM & 0.065 & 0.247 & 0.195 & 0.376 & 0.281 & 0.119 & 0.314 & 0.228 \\
\hline \multirow[t]{3}{*}{ Constraints } & 4-grams & 0.090 & 0.242 & 0.161 & 0.271 & 0.323 & 0.122 & 0.314 & 0.218 \\
\hline & WMT-19 best & $0.199 * * *$ & $0.346^{* * *} *$ & $0.306 * * *$ & $0.442 * * *$ & $\mathbf{0 . 3 8 0} * * *$ & $0.222 * * *$ & $0.431 * * *$ & 0.333 \\
\hline & & $\left(\mathrm{YISI}^{-1} 1_{\mathrm{SRL}}\right)$ & YISI-1 & (YISI-1) & $\left(\mathrm{YISI} 1_{\mathrm{SRL}}\right)$ & (YISI-1 SRL) & $\left(\mathrm{YISI} 1_{\mathrm{SRL}}\right)$ & $\left(\mathrm{YISI}^{-1} 1_{\mathrm{SRL}}\right)$ & \\
\hline
\end{tabular}

(b) Kendall's $\tau$ at the segment level

Table 7: WMT19 correlations of paraphrased BLEU for each method against human assessments (\# judgments in brackets) . Results that are significantly better than the sacreBLEU baseline are indicated as follows (at least $p \leq 0.05$ ) are marked in bold.

\begin{tabular}{llrrrrrr}
\hline \multirow{2}{*}{ Approach } & Method & Ave. & System & \multicolumn{3}{c}{ Segment } \\
\hline \multirow{2}{*}{ Baselines } & BEAM & 0.012 & 0.002 & 0.036 & 0.016 & 0.007 & 0.027 \\
$(+5)$ & RANDOM & 0.009 & 0.002 & 0.028 & 0.010 & 0.004 & 0.022 \\
& SAMPLED & 0.013 & 0.002 & 0.038 & 0.018 & 0.009 & 0.031 \\
\hline \multirow{2}{*}{ Diversity (+1) } & LASER & 0.009 & 0.002 & 0.025 & 0.011 & 0.005 & 0.017 \\
& TREELSTM & 0.009 & 0.001 & 0.025 & 0.011 & 0.004 & 0.019 \\
\hline \multirow{2}{*}{ Diversity (+5) } & LASER & 0.014 & 0.003 & 0.034 & 0.015 & 0.007 & 0.021 \\
& TREELSTM & 0.015 & 0.002 & 0.039 & 0.016 & 0.008 & 0.030 \\
\hline Output- & LASER & 0.007 & 0.000 & 0.020 & 0.009 & 0.003 & 0.018 \\
specific (+1) & TREELSTM & 0.010 & 0.002 & 0.020 & 0.013 & 0.004 & 0.021 \\
\hline Constraints & 4-grams & 0.004 & -0.050 & 0.027 & -0.002 & 0.043 & -0.084 \\
\hline
\end{tabular}

Table 8: Absolute gains in correlation for paraphrased Meteor for WMT19 with respect to the Meteor baseline. Significant gains (except for averages) are marked in bold $(p \leq 0.05)$. 


\begin{tabular}{llllllllll}
\hline \multirow{2}{*}{ Approach } & Method & $\begin{array}{l}\text { de-en } \\
(16)\end{array}$ & $\begin{array}{l}\text { fi-en } \\
(12)\end{array}$ & $\begin{array}{l}\text { gu-en } \\
(12)\end{array}$ & $\begin{array}{l}\text { kk-en } \\
(11)\end{array}$ & $\begin{array}{l}\text { lt-en } \\
(11)\end{array}$ & $\begin{array}{l}\text { ru-en } \\
(14)\end{array}$ & $\begin{array}{l}\text { zh-en } \\
(15\end{array}$ & Ave \\
\hline Baseline & METEOR & 0.909 & 0.993 & 0.883 & 0.969 & 0.972 & 0.825 & 0.941 & 0.927 \\
\hline \multirow{2}{*}{ Paraphrase } & BEAM & $\mathbf{0 . 9 2 7}$ & 0.994 & 0.887 & 0.976 & $\mathbf{0 . 9 8 3}$ & 0.862 & $\mathbf{0 . 9 4 9}$ & 0.940 \\
baselines (+5) & RANDOM & $\mathbf{0 . 9 2 0}$ & 0.994 & 0.889 & 0.974 & $\mathbf{0 . 9 8 1}$ & 0.853 & 0.945 & 0.937 \\
& SAMPLED & $\mathbf{0 . 9 2 5}$ & 0.995 & 0.891 & 0.978 & $\mathbf{0 . 9 8 2}$ & 0.864 & 0.945 & 0.940 \\
\hline \multirow{2}{*}{ Diversity (+1) } & LASER & $\mathbf{0 . 9 2 4}$ & 0.995 & 0.886 & $\mathbf{0 . 9 7 5}$ & $\mathbf{0 . 9 7 9}$ & 0.851 & $\mathbf{0 . 9 4 8}$ & 0.937 \\
& TREELSTM & $\mathbf{0 . 9 2 3}$ & 0.994 & 0.889 & 0.974 & $\mathbf{0 . 9 7 9}$ & 0.850 & $\mathbf{0 . 9 4 7}$ & 0.937 \\
\hline \multirow{2}{*}{ Diversity (+5) } & LASER & $\mathbf{0 . 9 3 2}$ & 0.995 & 0.890 & $\mathbf{0 . 9 7 8}$ & $\mathbf{0 . 9 8 3}$ & 0.860 & $\mathbf{0 . 9 5 0}$ & 0.941 \\
& TREELSTM & $\mathbf{0 . 9 3 0}$ & 0.995 & 0.894 & 0.977 & $\mathbf{0 . 9 8 3}$ & 0.864 & $\mathbf{0 . 9 5 0}$ & 0.942 \\
\hline Constraints & 4-grams & 0.922 & 0.990 & 0.910 & 0.983 & 0.988 & 0.775 & 0.949 & 0.931 \\
\hline & WMT-19 best & $\mathbf{0 . 9 5 0}$ & $\mathbf{0 . 9 9 5}$ & $\mathbf{0 . 9 9 3}$ & $\mathbf{0 . 9 9 8}$ & $\mathbf{0 . 9 8 9}$ & $\mathbf{0 . 9 7 9}$ & $\mathbf{0 . 9 8 8}$ & 0.985 \\
& & (YISI-1_SRL) & (METEOR) & (YISI-0) & (WMDO) & (ESIM) & (YISI-1) & (ESIM) & \\
\hline
\end{tabular}

(a) Pearson correlations at the system level.

\begin{tabular}{|c|c|c|c|c|c|c|c|c|c|}
\hline Approach & Method & $\begin{array}{l}\text { de-en } \\
(32000)\end{array}$ & $\begin{array}{l}\text { fi-en } \\
(23952)\end{array}$ & $\begin{array}{l}\text { gu-en } \\
(12192)\end{array}$ & $\begin{array}{l}\text { kk-en } \\
(11000)\end{array}$ & $\begin{array}{l}\text { lt-en } \\
(11000)\end{array}$ & $\begin{array}{l}\text { ru-en } \\
(28000)\end{array}$ & $\begin{array}{l}\text { zh-en } \\
(30000\end{array}$ & Ave \\
\hline Baseline & sentenceMETEOR & 0.061 & 0.243 & 0.197 & 0.356 & 0.275 & 0.145 & 0.351 & 0.233 \\
\hline \multirow{3}{*}{$\begin{array}{l}\text { Paraphrase } \\
\text { baselines }(+5)\end{array}$} & BEAM & 0.081 & 0.257 & 0.219 & 0.383 & 0.285 & 0.152 & 0.360 & 0.248 \\
\hline & RANDOM & 0.072 & 0.254 & 0.219 & 0.364 & 0.281 & 0.156 & 0.356 & 0.243 \\
\hline & SAMPLED & 0.080 & 0.262 & 0.228 & 0.375 & 0.292 & 0.160 & 0.360 & 0.251 \\
\hline \multirow{2}{*}{ Diversity (+1) } & LASER & 0.079 & 0.258 & 0.209 & 0.370 & 0.283 & 0.150 & 0.359 & 0.244 \\
\hline & TreeLstM & 0.074 & 0.255 & 0.210 & 0.374 & 0.284 & 0.149 & 0.357 & 0.243 \\
\hline \multirow{2}{*}{ Diversity $(+5)$} & LASER & 0.078 & 0.257 & 0.214 & 0.377 & 0.293 & 0.158 & 0.358 & 0.248 \\
\hline & TreeLstm & 0.074 & 0.259 & 0.228 & 0.378 & 0.287 & 0.153 & 0.361 & 0.249 \\
\hline \multirow[t]{3}{*}{ Constraints } & 4-grams & 0.098 & 0.237 & 0.193 & 0.272 & 0.318 & 0.145 & 0.351 & 0.230 \\
\hline & WMT-19 best & 0.20 & 0.35 & 0.31 & 0.44 & 0.38 & 0.22 & 0.43 & 0.333 \\
\hline & & $\left(\mathrm{YISI}{ }_{\mathrm{SRL}}\right)$ & (YISI-1) & (YISI-1) & $\left(\mathrm{YISI}{ }_{\mathrm{SRL}}\right)$ & $\left(\mathrm{YISI}{ }_{\mathrm{SRL}}\right)$ & $\left(\mathrm{YISI}{ }^{1} \mathrm{SRL}_{\mathrm{SR}}\right)$ & $\left(\mathrm{YISI}^{-1} \mathrm{SRL}_{\mathrm{SR}}\right)$ & \\
\hline
\end{tabular}

(b) Kendall's $\tau$ at the segment level

Table 9: WMT19 correlations of paraphrased METEOR for each method against human assessments (\# judgments in brackets) . Results that are significantly better than the METEOR baseline are indicated as follows (at least $p \leq 0.05$ ) are marked in bold. 


\begin{tabular}{l} 
Reference $\begin{array}{l}\text { Anyway the application phase for each of the } 20 \text { properties, which the municipality is offering at better conditions } \\
\text { and prices - similar to the earlier local model, has now ended. }\end{array}$ \\
\hline
\end{tabular}
and prices - similar to the earlier local model, has now ended.

LASER
prices, similar to the previous local model, is now over. However, the application phase for each of the 20 properties offered by the municipality at better conditions and prices, similar to the previous local model, is now over.

However, the application phase for each of the 20 properties offered by the municipality at better conditions and prices, similar to the previous local model, is now over.

However, the application phase for each of the 20 properties offered by the municipality at better conditions and TREELstm prices - similar to the previous local model - is now closed.

In any case, the application phase for each of the 20 properties offered by the municipality at better conditions and prices - similar to the previous local model - has now come to an end.

However, for each of the 20 properties that the municipality offers at better conditions and prices, similar to the previous local model, the application phase has now come to an end.

\begin{tabular}{|c|c|}
\hline Reference & And there is a need for discussion. \\
\hline LASER & $\begin{array}{l}\text { And we need to discuss it. } \\
\text { And we need to have a discussion. } \\
\text { And that's what we need to discuss. }\end{array}$ \\
\hline TreELstM & $\begin{array}{l}\text { And there's a need to discuss it. } \\
\text { And we need to discuss it. } \\
\text { It is necessary to discuss it. }\end{array}$ \\
\hline Reference & Why are there increasingly conflicts, despite successful integration? \\
\hline LASER & $\begin{array}{l}\text { Why is there ever more conflict, despite successful integration? } \\
\text { Why is there ever more conflict, despite successful integration? } \\
\text { Why are there more and more conflicts, despite the success of integration? }\end{array}$ \\
\hline TREELSTM & $\begin{array}{l}\text { Why is there more and more conflict, despite successful integration? } \\
\text { Why are conflicts growing despite successful integration? } \\
\text { Why are there ever more conflicts, despite the success of integration? }\end{array}$ \\
\hline Reference & Professor Doctor Aladin El-Mafaalani is standing before them. \\
\hline LASER & $\begin{array}{l}\text { In front of them is Professor Dr. Aladin El-Mafaalani. } \\
\text { Professor Dr. Aladdin El-Mafaalani stands in front of them. } \\
\text { Professor Dr. Aladdin El-Mafaalani stands in front of them. }\end{array}$ \\
\hline TREELSTM & $\begin{array}{l}\text { Professor Aladdin El-Mafaalani stands before them. } \\
\text { In front of them is Professor Aladdin El-Mafaalani. } \\
\text { There's Professor Aladdin El-Mafaalani in front of them. }\end{array}$ \\
\hline Reference & However, the operation took some time. \\
\hline LASER & $\begin{array}{l}\text { But the surgery took a while. } \\
\text { But the operation took a while. } \\
\text { But the surgery took a while. }\end{array}$ \\
\hline TREELSTM & $\begin{array}{l}\text { However, the surgery lasted a while. } \\
\text { But the surgery lasted a while. } \\
\text { However, it took some time to operate. }\end{array}$ \\
\hline Reference & $\begin{array}{l}\text { According to the police, it appears the fire was monitored for a long time, but the embers were inadvertently left } \\
\text { behind. }\end{array}$ \\
\hline LASER & $\begin{array}{l}\text { According to the police, the fire seems to have been under long surveillance, but the coals were inadvertently left } \\
\text { behind. } \\
\text { According to the police, the fire seems to have been under long surveillance, but the coals have been inadvertently } \\
\text { left behind. } \\
\text { According to the police, the fire seems to have been under long surveillance, but the coals were inadvertently left } \\
\text { behind. }\end{array}$ \\
\hline TREELSTM & $\begin{array}{l}\text { Police say the fire seems to have been under observation for a long time, but the coals are accidentally left behind. } \\
\text { The police seem to have been watching the fire for a long time, but the coals were accidentally left behind. } \\
\text { According to the police, the fire seems to have been under observation for a long time, but the coals were acciden- } \\
\text { tally left behind. }\end{array}$ \\
\hline Reference & What is the situation on the island now? \\
\hline LASER & $\begin{array}{l}\text { How's the island now? } \\
\text { What's happening on this island now? } \\
\text { What's the status on the island these days? }\end{array}$ \\
\hline TREELSTM & $\begin{array}{l}\text { What's it like on the island? } \\
\text { What's going on on the island? } \\
\text { So what's the status on the island? }\end{array}$ \\
\hline
\end{tabular}

Table 10: Top three paraphrases for seven sentences. 